\title{
Saliency of peripheral targets in gaze-contingent multiresolutional displays
}

\author{
EYAL M. REINGOLD \\ University of Toronto, Toronto, Ontario, Canada \\ and \\ LESTER C. LOSCHKY \\ University of Illinois at Urbana-Champaign, Champaign, Illinois
}

\begin{abstract}
Gaze-contingent multiresolutional displays (GCMRDs) have been proposed to solve the processing and bandwidth bottleneck in many single-user displays, by dynamically placing high-resolution in a window at the center of gaze, with lower resolution everywhere else. The three experiments reported here document a slowing of peripheral target acquisition associated with the presence of a gaze-contingent window. This window effect was shown for displays using either moving video or still images. The window effect was similar across a resolution-defined window condition and a luminance-defined window condition, suggesting that peripheral image degradation is not a prerequisite of this effect. The window effect was also unaffected by the type of window boundary used (sharp or blended). These results are interpreted in terms of an attentional bias resulting in a reduced saliency of peripheral targets due to increased competition from items within the window. We discuss the implications of the window effect for the study of natural scene perception and for human factors research related to GCMRDs.
\end{abstract}

Many new and proposed display technologies place tremendous demands on limited processing resources and transmission bandwidth. Such demands often involve various combinations of high image resolution, a large field of view, fast update rates, and low-bandwidth communication channels. Example applications include flight, driving, and medical simulators, immersive virtual reality (VR), remote piloting or driving, teleoperation, and videotelephony. Meeting the combined needs of such applications necessitates a reduction of processing resources and bandwidth. However, because all of the above-mentioned applications are single-user displays, a possible solution is to place high image resolution only at the point of gaze and lower resolution everywhere else. This requires dynamic updating of the high-resolution display area of interest, or window, whenever the gaze moves. The most natural method of achieving this is to use gaze-tracking technology. We will therefore refer to such displays as gaze-contingent multiresolutional displays (GCMRDs) (for a review, see Reingold, Loschky, McConkie, \& Stampe, in press). GCMRDs produce two

We gratefully acknowledge David Stampe for his invaluable assistance in programming the experiments and providing input throughout the project, and Steve Ito for data collection. Experiment 1 was previously presented at the Human-Computer Interaction International 2001 Conference, and Experiment 3 is to be presented at the Eye Tracking Research \& Applications Symposium 2002. Correspondence concerning this article should be addressed to E. M. Reingold, Department of Psychology, University of Toronto, 100 St. George Street, Toronto, ON M5S 3G3, Canada (e-mail: reingold@ psych.utoronto.ca). types of savings. First, given that information provided outside the high-resolution display area of interest is greatly reduced, there is a reduction in the bandwidth required for transmitting images. Second, under conditions in which images are being computer generated (e.g., immersive VR), the rendering requirements are reduced, because it is simpler to render low-resolution than highresolution image regions, and, therefore, there is a reduction in the need for computer processing resources (for specific illustrations of such savings, see Reingold et al., in press).

Whereas much work has been devoted to developing multiresolutional displays, often called variable-resolution, spatially variant resolution, area-of-interest, or regionof-interest displays (see Duchowski, 2002; Reingold et al., in press), far less work has been done to examine the effects that such displays have on the perception and performance of their users (but see Watson, Walker, Hodges, \& Worden, 1997, for work using head-contingent multiresolutional displays).

The present study is concerned particularly with the perceptual effects of GCMRDs. Previous research in this area has taken essentially two forms. The aim of the first line of research has been to find the set of display parameters that results in an imperceptible GCMRD - that is, one indistinguishable from a constant high-resolution display (Loschky, McConkie, Yang, \& Miller, 2002). However, such a display may not always be feasible, or even needed, for most applications. Thus, most GCMRD human factors research investigates the perception and performance effects produced by perceptible GCMRDs 
(i.e., displays with abnormalities that are quite perceptible to the user). The second line of work may therefore contribute to our understanding of the operation of the human visual system while laying the groundwork for selecting GCMRD system-design characteristics to achieve specified human performance goals.

The latter line of research has consistently found, for example, that "degrading" the visual periphery in GCMRDs results in shorter saccades (Loschky \& McConkie, 2000, 2002; Loschky et al., 2002; Shioiri \& Ikeda, 1989; van Diepen \& Wampers, 1998) and longer search times (Loschky \& McConkie, 2000, 2002; Parkhurst, Culurciello, \& Neibur, 2000; van Diepen \& Wampers, 1998). Loschky, McConkie, and colleagues (Loschky \& McConkie, 2002; Loschky et al., 2002) have shown that the shorter saccade lengths were due to a tendency to fixate more locations in the high-resolution area and fewer in the degraded area. They explained this as being due to a reduction in the salience of degraded peripheral saccade and search targets.

The present study was designed to further explore this hypothesis of reduced saliency for peripheral targets (i.e., for targets outside the window). Biresolutional displays were employed with higher resolution inside a gaze-contingent window and lower resolution outside the window. Such displays represent the most common type of GCMRDs and, consequently, it is important to investigate perception and performance issues related to this approach. Clearly, the discrete resolution drop-off method implemented in biresolutional displays doesn't match the continuous resolution drop-off function of the visual system. In particular, biresolutional displays introduce a relatively sharp resolution transition, or edges, into the visual field, which may produce perceptual problems. Thus, one of the questions investigated in the present study concerns whether a more gradual blending between the two resolution regions would attenuate the reduction in the salience of degraded peripheral saccade and search targets. In three experiments, we documented an interference effect hindering peripheral target acquisition in GCMRDs employing moving video (Experiments 1 and 2) or still images (Experiment 3 ). In addition, in the latter condition, sharp and blended window boundaries were contrasted, but this manipulation had no influence on the magnitude of the interference effect.

\section{EXPERIMENT 1}

In this experiment, a GCMRD with full-motion video was employed, and observers searched for a moving ring target. The present study posed the following question: If degraded objects in the visual periphery are less salient than those in the high-resolution window, will viewing a scene completely in low resolution make it easier to locate a salient peripheral target? Though counterintuitive, this might occur if objects in the high-resolution window competed for attention with the peripheral target, which would be less likely to happen when both the foveal and peripheral regions were degraded. To test this hypothesis, we compared four display conditions: (1) all lower resolution, (2) a small window, (3) a large window, and (4) all higher resolution. The dependent variables of interest were initial saccadic latency and total target acquisition time.

\section{Method}

Participants. The participants were 18 undergraduate students at the University of Toronto, who were paid for participating. All had normal or corrected-to-normal vision and were naive as to the purpose of the experiment.

Stimuli and Design. The stimuli were full-color video clips shot from a helicopter flying over landscapes (desert and canyon) containing a target moving against a moving background. The clips were each approximately $3 \mathrm{sec}$ long, shown at a rate of $30 \mathrm{frames} / \mathrm{sec}$ at $320 \times 240$ pixels. The average luminance was about $60 \mathrm{fL}$. There were two versions of each clip: filtered and unfiltered. The unfiltered video clips had an effective resolution of about $11 \mathrm{arc}$ min/line pair and an average luminance of about $60 \mathrm{fL}$. The filtered video clips were produced using a process equivalent to that of a Gaussian filter ( 0.5 cycles $/ \mathrm{deg}$, or cpd) to filter both the target and the background. The effective resolution of the filtered video clips was about 85 arc $\mathrm{min} /$ line pair using a $-6-\mathrm{dB}$ criterion, and the luminance was unchanged. In the filtered images, the target was also filtered, but still discriminable from the background, though sometimes only by target motion.

Resolution-defined windows were created by combining the filtered and unfiltered versions of the same clip, running simultaneously and synchronized in time. The unfiltered version of the video clip was displayed inside the window, and the blurred version of the video clip was displayed outside the window. We manipulated the size of the high-resolution circular window, with all other regions blurred. There were four display conditions: (1) filtered no window, (2) filtered small window (1.5 $5^{\circ}$ radius), (3) filtered large window ( $3^{\circ}$ radius), and (4) unfiltered no window (i.e., all higher resolution). Note that in the two window conditions, the level of low-pass filtering $(0.5 \mathrm{cpd})$ removes a large amount of higher spatial-frequency information that would otherwise be perceptible in much of the visual periphery (see Yang, Coia, \& Miller, 2001). Thus, the filtering should produce highly noticeable image degradation. All the windows were centered at the participant's gaze position, as measured by the EyeLink gaze tracking system (described below). The edges of the window remained sharp; there was no blending region between the window and the background.

The target, a $1^{\circ}$ ring, moved in a straight line at a constant velocity of approximately $8 \% \mathrm{sec}$ from the beginning to the end of the video clip. The color of the target was selected by averaging the color of the background on which it appeared, and the target's luminance was raised by $40 \%$ to $80 \%$ relative to the background. This coloring technique was designed to make target search dependent on the motion of the target (i.e., it would be diff icult to discriminate the target from the background in a static scene). There were four directions of target motion: vertically down the left side, vertically down the right side, diagonally down and to the left, and diagonally down and to the right. The backgrounds were 16 video clips of mountainous and desert terrains shot from a moving helicopter, some from a forward-looking vantage point, which contained opticflow cues for forward self-motion, and some from directly above and looking down. All background motion was from the top to the bottom of the screen, but never in the same direction or at the same speed as the target motion.

Apparatus. The SR Research Ltd. EyeLink eye-tracking system used in this research has high spatial resolution $\left(0.01^{\circ}\right)$ and a sampling rate of $250 \mathrm{~Hz}$ (4 msec temporal resolution). The three cameras on the EyeLink headband allow simultaneous tracking of both 
eyes and of head position, computing true gaze position with unrestrained head motion. Only the participant's dominant eye was tracked in these studies. The EyeLink system uses an Ethernet link between the eye tracker and display computers to supply real-time gaze position and saccade event data. The on-line saccade detector of the eye tracker was set to detect saccades with an amplitude of $0.5^{\circ}$ or greater, using an acceleration threshold of $9,500^{\circ} / \mathrm{sec}^{2}$ and a velocity threshold of $30 \% \mathrm{sec}$. Two additional computers, both $66-\mathrm{MHz} 486-\mathrm{DX}$ PC compatible, were used to concurrently play the processed (filtered or high-luminance) and unprocessed video clips, respectively, and to feed the video to the display computer. The display computer, a 100-MHz 486-DX PC compatible, controlled stimulus presentation, integrated incoming video signals, and displayed one channel as background imagery and part of the other channel as a window at the participant's point of gaze on a 17-in. ViewSonic 17PS monitor. The display was positioned at a viewing distance of $60 \mathrm{~cm}$ so that the total field of view was $30^{\circ}$ (horizontal) $\times 24^{\circ}$ (vertical). The total system throughput delay (the time it takes from the eye movement to a change in the display) was $21 \mathrm{msec}$.

Procedure. The task for the participants was to acquire (i.e., look directly at) a target as rapidly as possible and track it until the video clip ended. No other response was needed. A trial sequence began with a fixation dot on a blank screen. The participant fixated the dot, and the experimenter initiated the trial when the gaze cursor stabilized. The fixation dot disappeared, and after approximately $0.5 \mathrm{sec}$ the video clip started. When the video clip ended, the fixation dot reappeared, and the next trial began. The participants received a practice block of 8 trials, followed by four experimental blocks of 16 trials each, for a total of 64 trials per subject. Each block contained 4 trials for each of the four display conditions (filtered no window, small window, large window, and unfiltered). In addition to measuring target acquisition time, the participants' subjective impressions of image quality were collected.

A 9-point calibration, followed by a 9-point calibration accuracy test, was performed at the start of the experiment. Calibration was repeated if the error at any point was more than $1^{\circ}$, or if the average error for all points was greater than $0.5^{\circ}$. Before each trial, a black fixation target was presented at the center of the display. The participant fixated this target and the gaze position measured during the fixation was used to correct any postcalibration drift errors. Throughout each trial, the experimenter was able to view on a separate monitor the target path, overlaid with a cursor corresponding to real-time gaze position. If the experimenter judged that gazetracking accuracy had declined, he initiated a full calibration before the next trial. However, this occurred very infrequently.

\section{Results and Discussion}

Subjectively, the participants reported that the filtering produced very noticeable peripheral image degradation. Figure 1 summarizes task performance in each of the four experimental conditions by plotting average tracking error (distance of gaze from the target) during the first $1,000 \mathrm{msec}$ of clip viewing. As is shown in the figure, the unfiltered no-window condition produced better performance than did the filtered no-window condition, and both of these conditions resulted in better performance than did either the small or the large window condition (performance did not differ across the two window conditions).

We used two dependent measures to quantify performance: initial saccadic latency and target acquisition time. For the purposes of the analyses, a saccade was defined as any eye movement with a peak velocity over a threshold of $25 \% \mathrm{sec}$ and an amplitude of at least $1^{\circ}$. The latency to first saccade (i.e., initial saccadic latency) was the time from video onset to the first saccade (in any direction). The latency to acquisition was the time from the start of the video clip (video onset) to acquisition of the target, defined as the first full 20-msec period in which the gaze position remained within $2^{\circ}$ of the target. Not included in the analyses were (1) trials containing a blink during the period beginning $100 \mathrm{msec}$ prior to video onset and ending $80 \mathrm{msec}$ following target acquisition, (2) trials in which acquisition occurred more than $2 \mathrm{sec}$

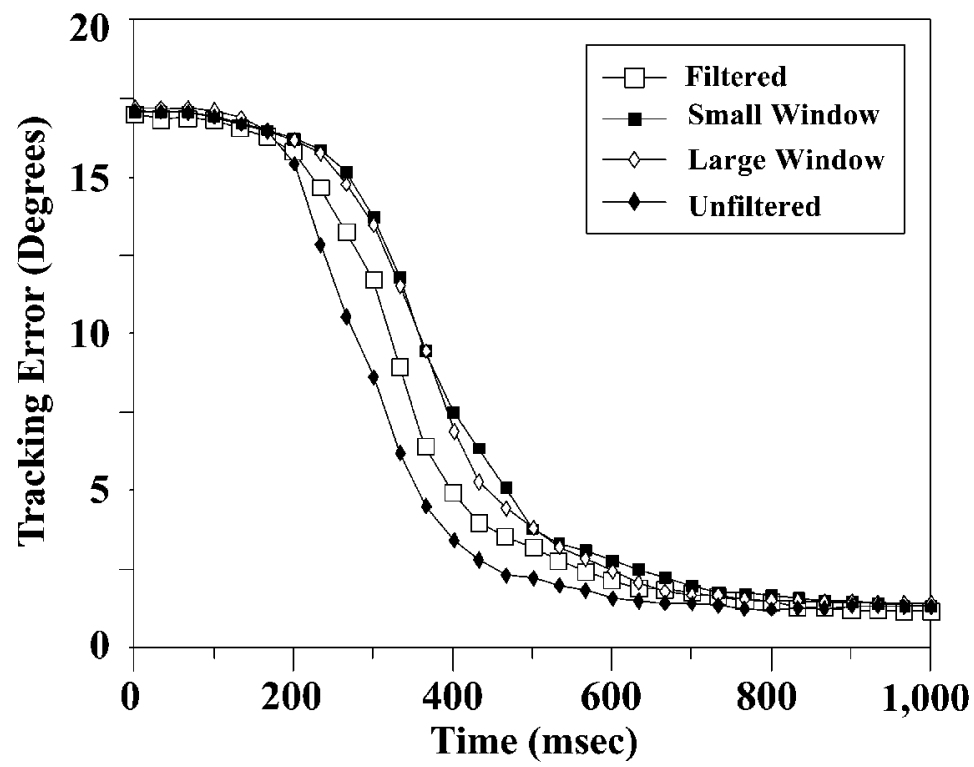

Figure 1. Distance of gaze from the target during the first $1,000 \mathrm{msec}$ of clip viewing in Experiment 1. 
following video onset, (3) trials in which error was greater than $3^{\circ}$ in the first $100 \mathrm{msec}$ after acquisition, (4) trials in which the average error was greater than $2^{\circ}$ after acquisition, and (5) trials in which anticipatory saccades - that is, any saccades made within $100 \mathrm{msec}$ before or $60 \mathrm{msec}$ after video onset-were made. In total, $6.1 \%$ of the trials were excluded.

As is shown in Figure 2, for both dependent measures, whereas performance in the unfiltered no-window condition was clearly the best (all $t \mathrm{~s}>9.71, p<.001$ ), the filtered no-window condition resulted in better performance than did either the small or the large window condition (all $t \mathrm{~s}>3.37, p<.01)$. Performance did not significantly differ across the two window conditions (all $t \mathrm{~s}<1$ ).

The results of the experiment were rather counterintuitive, since more visual information resulted in poorer target-detection performance. Specifically, it is clear that the biresolutional displays (i.e., gaze-contingent window conditions) led to inferior performance in comparison with the filtered no-window (i.e., all low-pass filtered, low-resolution) condition. Longer initial saccadic latencies in the window conditions were observed, and this initial slowing was likely responsible for the longer total target acquisition times. This is the case because the magnitude of the window effects on initial latencies and acquisition times were similar. Moreover, across participants, the correlation between these two measures reveals that, on average, $62.3 \%$ of the variance in acquisition times can be accounted for by the variance in initial saccadic latencies.

These findings are consistent with the hypothesis that the window conditions reduce the relative salience of the peripheral targets. However, it is unclear whether the filtering of the peripheral image in the window conditions (i.e., lower resolution outside than inside the window) was a prerequisite for producing the reduction in the salience of the peripheral targets. In other words, it is important to distinguish between the effects of filtering and those of windowing. In order to accomplish that, in the next experiment, we introduced a gaze-contingent window condition that did not involve degrading the image outside the window. This would allow for the possibility of showing an effect of a gaze-contingent window on the detection of peripheral targets in the absence of peripheral image filtering.

\section{EXPERIMENT 2}

In this experiment, two types of window were used: the standard resolution-defined window, with higher resolution in the window and a low-pass filtered periphery, and a luminance-defined window in which the luminance inside the window was increased by $20 \%$ and luminance outside the window was unchanged. Note that in the luminance-defined window condition, a window was present, but the quality of the peripheral image was preserved (i.e., it was the same as the all-higher-resolution condition). Two no-window conditions, one in which the display is uniformly higher resolution and one in which the display is uniformly low-pass filtered, were used as well. As in Experiment 1, the task was to detect peripheral target stimuli moving across the screen. It was hypothesized that the presence of both types of windows, resolution defined and luminance defined, would impair performance on this task, indicating that the effect of a salient window can impair peripheral task performance independently of resolution differences between the central and peripheral regions of the image. Furthermore, it was hypothesized that the fil-

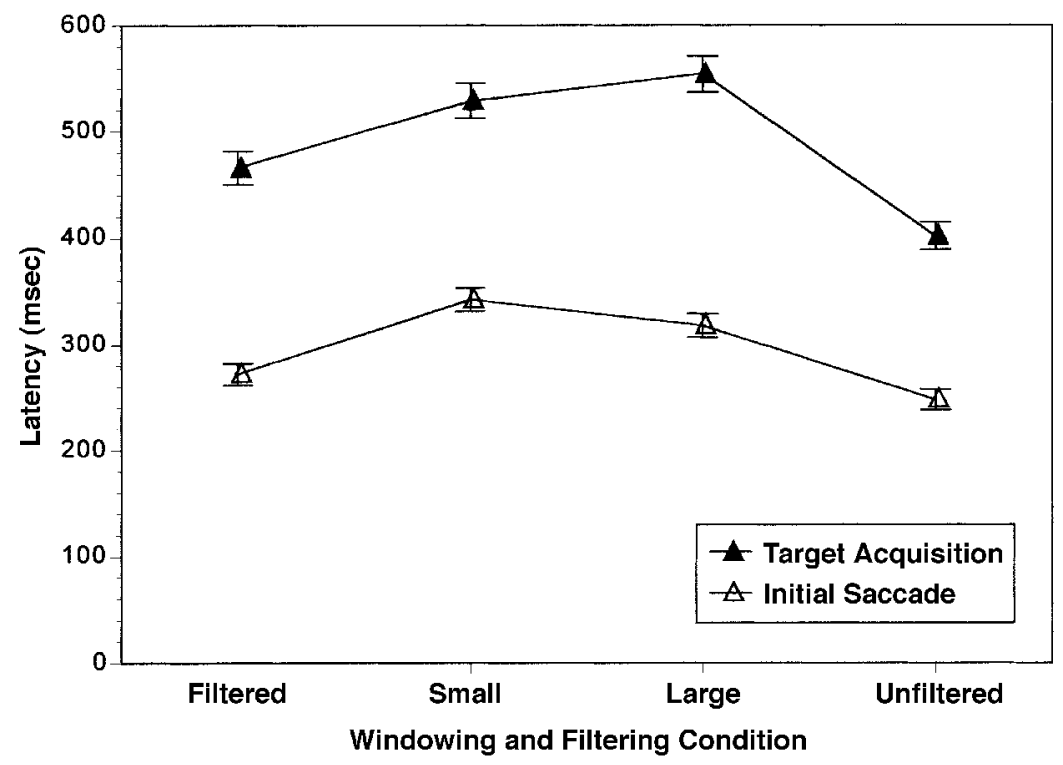

Figure 2. Initial saccadic latency and target acquisition latency, in milliseconds, as a function of the four filtering conditions of Experiment 1. 
tering of the periphery in both the window-present and the window-absent conditions would impair performance, indicating that the degraded quality of the peripheral imagery also impairs detection performance.

\section{Method}

Participants. The participants were 60 undergraduate students at the University of Toronto, who received credit in an introductory psychology course for participation. All had normal or correctedto-normal vision and were naive as to the purpose of the experiment.

Design. A $2 \times 2$ (filtering $\times$ windowing) design was used. There were two levels of filtering, filtered and unfiltered, and two levels of windowing, window and no window, for a total of four conditions. The filtered window condition was the previously described resolution-defined window condition, whereas the unfiltered window condition was the luminance-defined window condition. In the filtered no-window condition, the image was uniformly low-pass filtered, and in the unfiltered no-window condition, the image was uniformly higher resolution.

The four conditions were counterbalanced with the four types of target motion for a total of 16 combinations. Each combination and each background scene appeared in a random sequence four times per block, for a total of 64 trials per block. Three blocks of trials were used in the experiment. Before the experiment began, the participants were given a practice block of eight trials.

Stimuli. The stimuli were identical to those used in Experiment 1 , with the following exceptions. All the windows were roughly circular with a $3^{\circ}$ radius. Luminance-defi ned windows were created by displaying the unfiltered version of the video clip across the entire screen, but selectively increasing the luminance inside the window by $20 \%$.

Apparatus and Procedure. The apparatus and procedure were identical to those used in Experiment 1.

\section{Results and Discussion}

We used the same exclusion criteria for the data as those used in Experiment 1, and a total of 5.8\% of the trials were dropped. As in Experiment 1, we measured the partici- pants' initial saccadic latency and target acquisition latency. Figure 3 illustrates that initial saccadic latencies and acquisition times were slowed by the presence of a window, and that this was true for both the resolutiondefined window and the luminance-defined window.

To further explore the effects of windowing and filtering on task performance, $2 \times 2$ (filtering $\times$ windowing) within-subjects analyses of variance (ANOVAs) were conducted, with initial and second saccadic latency, acquisition time, number and amplitude of saccades prior to target acquisition, and first-saccade error as dependent variables. The results of these analyses are summarized in Table 1. As can be seen in the table, both filtering and windowing had a negative impact on task performance. Specifically, both filtering and windowing produced longer initial saccadic latencies and acquisition times, more saccades, and shorter saccadic amplitudes. In addition, filtering, but not windowing, also resulted in greater first-saccade error (i.e., gaze-to-target distance) and longer second-saccade latency (i.e., longer first-fixation duration). The only significant filtering $\times$ windowing interaction reflected greater reduction in saccadic amplitude caused by the resolution-defined window $\left(1.0^{\circ}\right)$ than by the luminance-defined window $\left(0.6^{\circ}\right)$.

The results of this study show that both the presence of a window and low-pass filtering of the peripheral target increase the time taken to initiate the first saccade to a peripheral target and to acquire that target. As was argued earlier, the slowing of initial saccadic latency most likely mediates the longer total target acquisition times. This conclusion is consistent with the present finding that, on trials in which more than a single saccade is executed prior to target acquisition, the latency of the second saccade is unaffected by the windowing manipulation.

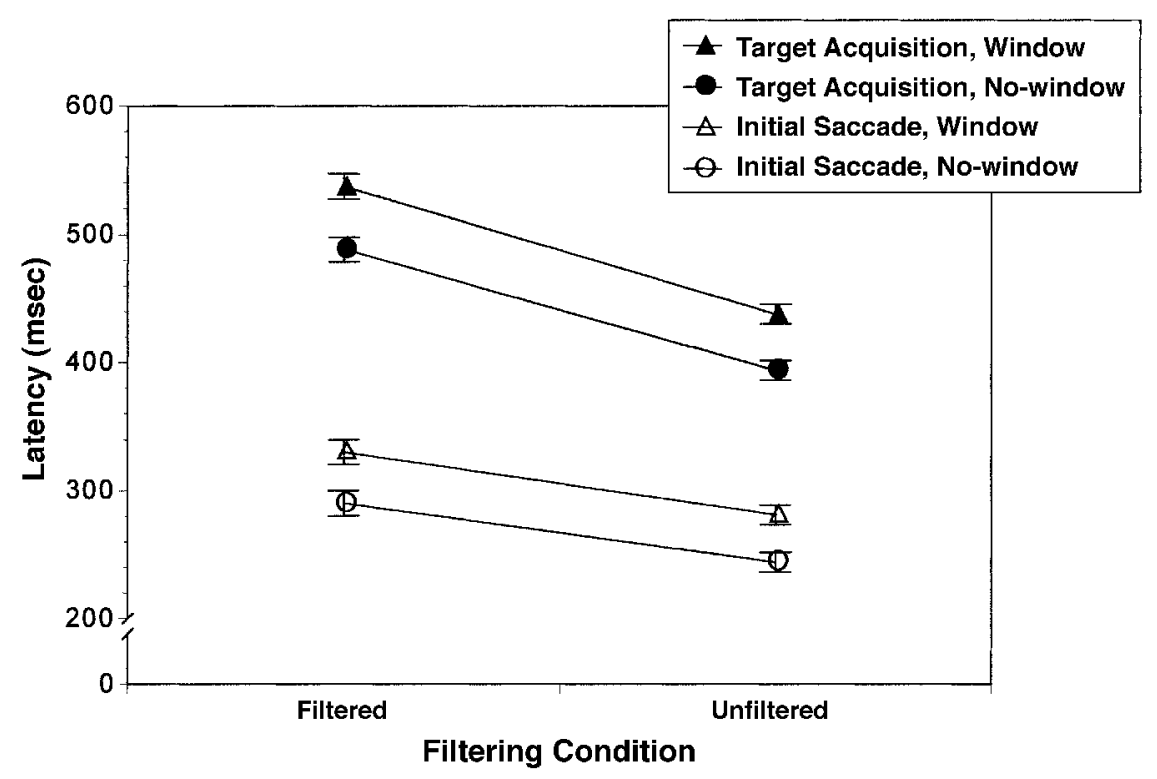

Figure 3. Initial saccadic latency and target acquisition latency, in milliseconds, as a function of the windowing and filtering conditions of Experiment 2. 
Table 1

Effects of Windowing and Filtering on Mean Initial and Second Saccadic Latency, Acquisition Time, Number and Amplitude of Saccades Prior to Target Acquisition, and First-Saccade Error in Experiment 2

\begin{tabular}{|c|c|c|c|c|c|c|c|c|c|c|c|}
\hline \multirow[b]{3}{*}{ Dependent Variable } & \multicolumn{4}{|c|}{ Filtered } & \multicolumn{4}{|c|}{ Unfiltered } & \multirow{3}{*}{$\begin{array}{c}\begin{array}{c}\text { Windowing } \\
\text { Effect }\end{array} \\
M\end{array}$} & \multirow{3}{*}{$\begin{array}{c}\begin{array}{c}\text { Filtering } \\
\text { Effect }\end{array} \\
M\end{array}$} & \multirow[b]{3}{*}{ Interaction } \\
\hline & \multicolumn{2}{|c|}{ Window } & \multicolumn{2}{|c|}{ No Window } & \multicolumn{2}{|c|}{ Window } & \multicolumn{2}{|c|}{ No Window } & & & \\
\hline & $M$ & $S E$ & $M$ & $S E$ & $M$ & $S E$ & $M$ & $S E$ & & & \\
\hline Initial saccade latency (msec) & 343 & 7.6 & 305 & 6.6 & 291 & 6.7 & 253 & 6.8 & $\begin{array}{c}38 \\
p<.001\end{array}$ & $\begin{array}{c}52 \\
p<.001\end{array}$ & $\begin{array}{c}\text { n.s.; } \\
p=.93\end{array}$ \\
\hline $\begin{array}{l}\text { Second-saccade latency (msec) } \\
\text { (First-fixation duration) }\end{array}$ & 162 & 7.0 & 157 & 8.5 & 136 & 6.7 & 131 & 4.9 & $\begin{array}{c}5 \\
\text { n.s.; } p=.36\end{array}$ & $\begin{array}{c}26 \\
p<.001\end{array}$ & $\begin{array}{c}\text { n.s.; } \\
p=.67\end{array}$ \\
\hline Acquisition time (msec) & 538 & 10.4 & 481 & 9.9 & 435 & 9.0 & 392 & 7.7 & $\begin{array}{c}50 \\
p<.001\end{array}$ & $\begin{array}{c}96 \\
p<.001\end{array}$ & $\begin{array}{c}\text { n.s.; } \\
p=.10\end{array}$ \\
\hline Number of saccades & 1.60 & .04 & 1.52 & .04 & 1.42 & .03 & 1.40 & .03 & $\begin{array}{c}0.05 \\
p<.05\end{array}$ & $\begin{array}{c}0.15 \\
p<.001\end{array}$ & $\begin{array}{c}\text { n.s.; } \\
p=.08\end{array}$ \\
\hline Amplitude of saccades (deg) & 8.84 & .23 & 9.84 & .22 & 10.06 & .21 & 10.62 & .18 & $\begin{array}{c}-0.78 \\
p<.001\end{array}$ & $\begin{array}{c}-1.00 \\
p<.001\end{array}$ & $p<.05$ \\
\hline First-saccade error (deg) & 4.25 & .26 & 4.00 & .25 & 3.36 & .20 & 3.50 & .20 & $\begin{array}{c}0.06 \\
\text { n.s.; } p=.76\end{array}$ & $\begin{array}{c}0.70 \\
p<.001\end{array}$ & $\begin{array}{c}\text { n.s.; } \\
p=.07\end{array}$ \\
\hline
\end{tabular}

Note-Windowing effects $=$ average performance in window conditions minus average performance in no-window conditions; filtering effects $=$ average performance in filtered conditions minus average performance in unfiltered conditions.

The present findings of longer target acquisition times and shorter saccades in the window conditions are also consistent with the results from studies documenting shorter saccade lengths and longer search times with GCMRDs having highly degraded peripheries (Loschky \& McConkie, 2000, 2002; Loschky et al., 2002; Parkhurst et al., 2000; Shioiri \& Ikeda, 1989; van Diepen \& Wampers, 1998). By distinguishing the effects of windowing and low-pass filtering, the present study suggests that peripheral target detection with GCMRDs may be negatively impacted not only by the loss of perceptual detail due to filtering, but also by the mere presence of the gaze-contingent window. Indeed, for some of the measures we employed, the windowing effect appears to be just as strong using a luminance-defined window as a resolution-defined window. This strengthens the argument that the windowing effect is due to greater relative salience of objects within the window in comparison to those outside it, including the target.

Nevertheless, there is an alternative explanation for the results of Experiments 1 and 2. Specifically, the window conditions employed involved a sharp boundary between the regions inside and outside the window due to the resolution or luminance difference across these display areas. Consequently, the salience of the window boundary might be able to explain the longer initial saccadic latencies in Experiments 1 and 2. If the window boundary is salient, it might compete with the target for attention when the display initially appears on the screen, thus resulting in the longer initial saccadic latencies found in both experiments. Unfortunately, neither Experiment 1 nor Experiment 2 provided any way of distinguishing whether, relative to the target in the periphery, it is the objects in the window that are salient or the window boundary that is salient.

Questions regarding the impact of the window boundary on the perception and performance of observers in GCMRDs are also important for applied reasons. Specif- ically, when biresolutional displays have been used in flight simulators, it has frequently been reported that users prefer larger windows, because with smaller windows the edges are more visible (see, e.g., Turner, 1984). If the findings of Experiments 1 and 2 were shown to be due to the visibility of the boundary of the window, this would add further support to the claim that designers of GCMRD applications should avoid having such boundaries.

\section{EXPERIMENT 3}

In this experiment, we had two chief goals. First, we wanted to test the hypothesis that a sharp boundary is necessary to produce the windowing effect of Experiments 1 and 2-that is, a slowing of initial saccadic latencies to a salient peripheral target in the biresolutional condition relative to an all-low-pass condition. In order to test this hypothesis, we decided to compare window conditions in which there was either a sharp or a smooth resolution boundary. If we were to find that initial saccadic latencies are longer in both the sharp and the smooth-boundary window conditions than in an all-lowpass filtered condition, as in both Experiments 1 and 2, this would add strength to the argument that visual salience is reduced outside the window. If the window effect disappears when the window boundary is smooth, then this would suggest that sharp boundaries are generally problematic for perception in GCMRDs.

Second, we wanted to see if we could replicate the windowing effect of Experiments 1 and 2 with a GCMRD using static images. Since both of the above experiments used full-motion video, it is possible that the windowing effects found in those experiments are limited to moving targets and/or a moving image context. Thus, we decided to use a GCMRD with static images and static targets. If the windowing effect from the previous two experiments generalizes to the static targets and scene contexts, this 
would suggest that more general perceptual processes are involved in the effect, and that image motion is not a necessary component of it.

\section{Method}

Participants. The participants were 45 undergraduate students at the University of Toronto, who were paid for participating. All had normal or corrected-to-normal vision and were naive as to the purpose of the experiment.

Stimuli and Design. The images used were 72 images of residential interiors. The image size was $360 \times 240$ pixels, and the display subtended $30^{\circ} \times 24^{\circ}$, filling the entire screen, for resolutions of 12 pixels per degree horizontally and 10.7 pixels per degree vertically. One target - a $7 \times 7$ pixel (about $0.6^{\circ}$ ) white cross with a black border - was added to each image. The targets were placed on one of the four diagonals, at a distance of $12^{\circ}$ from the central fixation point. For each of the 288 image $(72) \times$ target-location (4) combinations, filtered versions were created by using a Gaussian low-pass filter of $1.0 \mathrm{cpd}$.

On some trials, a $12^{\circ}$ square window was dynamically centered on the participant's point of gaze (i.e., the edge of the window was $6^{\circ}$ from the center of vision vertically or horizontally; see Figure 4 ). Within the window, the image was relatively high resolution (i.e., as in the unfiltered image). Outside the window, the image was in lower resolution (i.e., as in the filtered image). Three window display conditions were used: the filtered no-window condition (the entire image was uniformly low-pass filtered), the sharp-boundary window condition (a $12^{\circ}$ window with no blending region), and the blended-boundary window condition (a $12^{\circ}$ window with a $3^{\circ}$ wide blending region). In the latter condition, a blending function was used at the edges of the window to mix periphery (filtered) and foreground (unfiltered) images, with the ratio changing linearly. For example, moving up, down, left, or right from the participant's point of gaze, the image was at full resolution up to $4.5^{\circ}$ from the participant's point of gaze, was a $50 \%$ mix of the full-resolution and lower-resolution images at $6^{\circ}$, and was all at lower resolution past $7.5^{\circ}$. It is important to note that, as in Experiments 1 and 2, the degree of low-pass filtering used in this experiment $(1 \mathrm{cpd})$ reduced image resolution outside the window well below the sensitivity limits of the human visual system for much of the visual periphery (Loschky et al., 2002; Yang et al., 2001). Thus, the filtering should have produced very noticeable image degradation. In the blendedboundary window condition, the participants reported that they were aware that parts of the image were degraded but that they were unable to perceive the blend (i.e., they perceived smooth degradation into the periphery). In contrast, in the sharp-boundary window condition, the participants reported perceiving the contours of the window as an abrupt change in the quality of the image.

Each participant performed in 12 blocks of 72 trials. Across blocks, each of the 288 image $\times$ target-location combinations appeared once in each of the three window conditions (filtered no window, sharp-boundary window, and blended-boundary window), for a total of 864 trials in the experiment.

Apparatus. The eyetracker and monitor were the same as those used in Experiments 1 and 2. The display was generated using an S3 VGA card, and the frame rate was $120 \mathrm{~Hz}$. The average delay between an eye movement and the update of the gaze-contingent window was 14 msec.

Procedure. The procedure was identical to that used in Experiment 1 .

\section{Results and Discussion}

Custom analysis software was used to process the eye movement data files. Trials were rejected because of anticipation if the participant made a substantial saccade (more than $2^{\circ}$ ) or a blink either before the picture was presented or less than 70 msec after its appearance. Trials were also rejected if the first saccade made by the participant was less than $3^{\circ}$ in magnitude, or if the directional error of the first saccade was more than $22.5^{\circ}$. These exclusions accounted for $2.2 \%$ and $3.4 \%$ of the total trials, respectively. Analyses were then performed on the remaining trials. The results show that the target was generally quite salient, with the initial saccade endpoint falling within $3^{\circ}$ or less of the target on $86 \%$ of all trials. Consequently, the best measure of acquisition speed was deemed to be the initial saccadic latency measure.

As is shown in Table 2, the all-low-pass filtered nowindow condition produced reliably shorter mean initial saccadic latencies to the target than did either of the window conditions [no window vs. sharp boundary, $t(44)=7.13$, $p<.001$; no window vs. blended boundary, $t(44)=6.87$, $p<.001]$, whereas latencies in the sharp- and blendedboundary windows were identical to each other.

The results of Experiment 3 suggest that having a gaze-contingent window results in longer initial saccadic latencies than does an all-low-pass filtered image, but whether the gaze-contingent window boundary is sharply defined or smoothly gradated makes no difference. This allows us to reject the window-boundary-artifact explanation of our results. This strengthens the argument that the objects inside the window become relatively more salient than they otherwise would have been, resulting in increased competition for attention between objects in the high-resolution window and the target in the periphery.

The results of Experiment 3 also show that the slowing of initial saccadic latencies in windowed conditions is a robust effect and is not dependent on using full-motion video as in Experiments 1 and 2. However, the 14-msec windowing effect in the present experiment (see Table 2) was smaller than the windowing effects in Experiments 1 and 2, which were $58 \mathrm{msec}$ and $40 \mathrm{msec}$, respectively. Whether this difference in the size of the windowing effect was due to the full-motion versus the still-image factor, or to some other difference between these studies (e.g., salience of the target vs. the periphery, color vs. monochrome images, etc.) will need to be determined by further research.

\section{GENERAL DISCUSSION}

In this study, we demonstrated that programming a saccade to a peripheral target can be disrupted by the presence of a gaze-contingent window. We demonstrated that

Table 2

Effect of Window Type on Mean Initial Saccadic Latency in Experiment 3

\begin{tabular}{lcc}
\hline & \multicolumn{2}{c}{ Initial Saccadic Latency $(\mathrm{msec})$} \\
\cline { 2 - 3 } Window Type & $M$ & $S E$ \\
\hline All low-pass, None & 191 & 3.9 \\
Sharp boundary & 205 & 4.3 \\
Blended boundary & 205 & 4.2 \\
\hline
\end{tabular}


this windowing effect was obtained regardless of whether or not peripheral degradation or filtering is used (Experiment 2) and when either sharp or smooth window boundaries were employed (Experiment 3 ). This effect appears to be quite general and was obtained with either moving video (Experiments 1 and 2) or still images (Experiment 3 ).

We propose an account of the windowing effect in terms of attentional factors. Specifically, we hypothesize a type of attentional capture caused by the gaze-contingent window, reflecting an increase in saliency of objects inside the window, and a relative decrease in saliency for peripheral objects (see Itti \& Koch, 2001, for a review of computational approaches to visual salience). It is this increased competition between the objects in the window and the peripheral target that causes the windowing effect we observed.
Our effect is similar to other findings of interference with performance on peripheral detection tasks as a function of increased foveal load (Crundall, Underwood, \& Chapman, 1999; Holmes, Cohen, Haith, \& Morrison, 1977; Ikeda \& Takeuchi, 1975; Mackworth, 1965; Pomplun, Reingold, \& Shen, 2001; Williams, 1985, 1988, 1989; see Williams, 1988, for a review). For example, Holmes et al. (1977) demonstrated that the mere presence of a foveal item that subjects were instructed to ignore resulted in poorer peripheral task performance (see also Ikeda \& Takeuchi, 1975; Mackworth, 1965). Holmes et al. interpreted this finding as a general interference effect; the foveal item draws the attention of the observer and, thus, interferes with processing of other stimuli in the visual field. Given that this decline in peripheral task performance was sometimes found to be greater for tar-

A
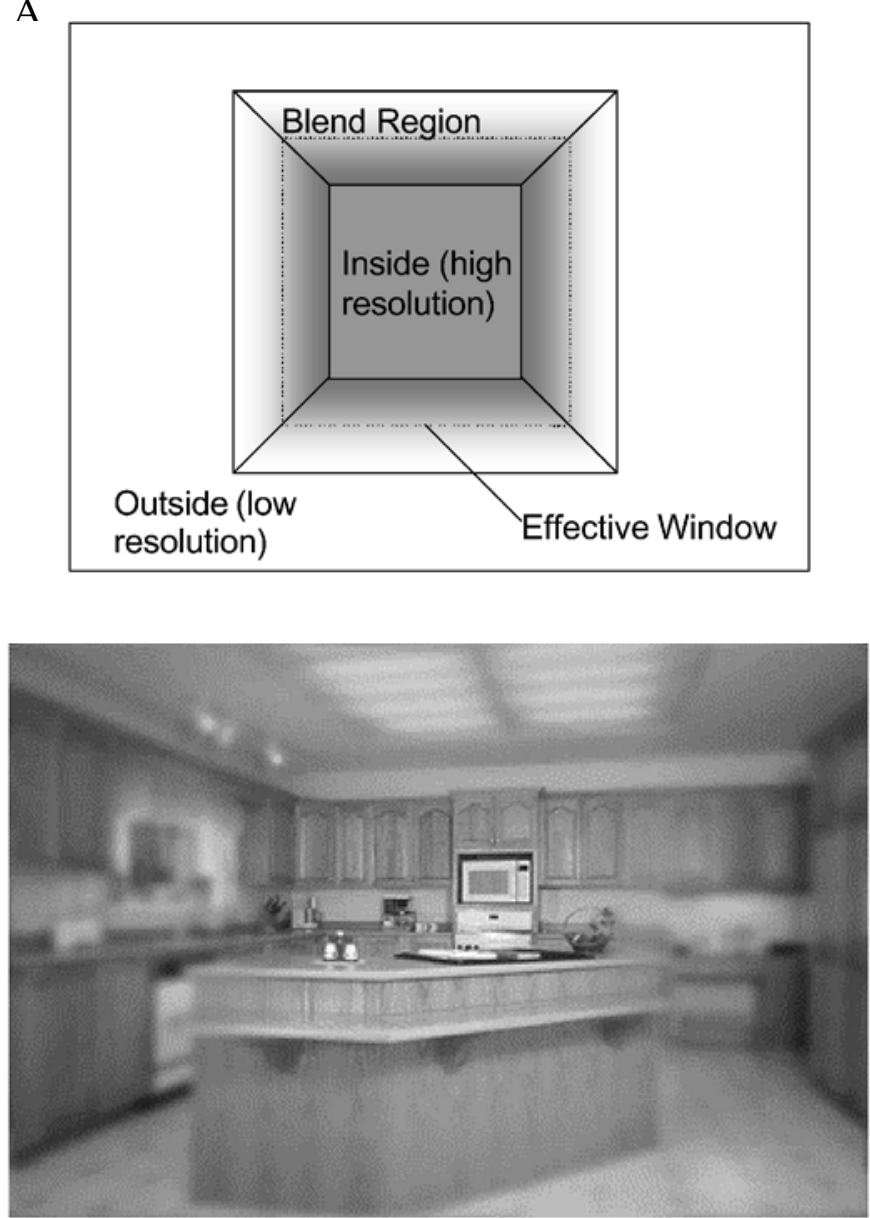

Figure 4. (A) A blended-boundary wind ow: The high-resolution area inside the window fades into the low-resolution background over several degrees. The mixture of foreground and background images varies linearly within the blending region. The effective window area is set to the center of the blending region. (B) An illustration of a $12^{\circ}$ blendedboundary window (the participant's gaze position is at the center of the screen). Filtering outside the window was produced by using a Gaussian low-pass filter of $1.0 \mathrm{cpd}$. 
gets at larger eccentricities (Mackworth, 1965; Williams, 1985), it was argued that the foveal load reduced the useful field of view, leading to the coining of the controversial term tunnel vision (see Williams, 1988).

Regardless of the mechanism responsible for the windowing effect we documented, this effect has important implications for human factors research related to GCMRDs. Taken together, the windowing effect and previous findings showing shorter saccade lengths and longer search times in GCMRDs with perceptible peripheral image degradation (Loschky \& McConkie, 2000, 2002; Loschky et al., 2002; Parkhurst et al., 2000; Shioiri \& Ikeda, 1989; van Diepen \& Wampers, 1998) clearly point to perception and performance costs that may be associated with the use of such GCMRDs.

However, the practical implications of such effects should vary greatly depending on the specific area of application. In piloting situations, split-second delays in reacting to peripheral stimuli-for example, a missile flarecan have severe consequences. But there may be no important consequences for such a delay in video-telephony or Internet image download applications. Likewise, we would expect that under conditions in which it is important to identify a target or to discriminate it from nontargets, the window conditions would undoubtedly be superior to the filtered condition in which considerable high spatial-frequency information was lost (i.e., no details could be seen). Therefore, the windowing effect we have shown may be specific to the task of orienting to salient peripheral targets. Moreover, recently, Loschky et al. (2002) have shown that it is possible to construct GCRMDs having substantial image filtering without any discernible perception and performance costs. Unlike the biresolutional displays used in the current study, Loschky et al. (2002) employed continuous resolution drop-off functions derived from psychophysical studies of visual sensitivity (Yang et al., 2001).

It is also noteworthy that the current results showed no effect of blending the boundary between levels of resolution. This therefore fails to support the claim that such blending is important in GCMRDs. However, given the limited nature of the present analyses, it would be premature to draw strong conclusions based on this null result. Clearly, more research is required in order to investigate the perceptual and attentional factors underlying the windowing effect documented here. Nevertheless, our preliminary findings indicate that this effect may have important implications for both applied and basic investigations of eye movements during the performance of complex naturalistic tasks.

\section{REFERENCES}

Crundall, D. E., Underwood, G., \& Chapman, P. R. (1999). Driving experience and the functional field of view. Perception, 28, 10751087.

Duchowski, A. T. (2002). A breadth-first survey of eye-tracking applications. Behavior Research Methods, Instruments, \& Computers, 34, 455-470.

Holmes, D. L., Cohen, K. M., Haith, M. M., \& Morrison, F. J. (1977). Peripheral visual processing. Perception \& Psychophysics, 22, 571-577.

IKEDA, M., \& TAKEUCHI, T. (1975). Influence of foveal load on the functional visual field. Perception \& Psychophysics, 18, 255-260.

IтTI, L., \& Косн, C. (2001). Computational modeling of visual attention. Nature Reviews Neuroscience, 2, 194-203.

LoschKy, L. C., \& McConKIE, G. W. (2000). User performance with gaze contingent multiresolutional displays. In A. T. Duchowski (Ed.), Proceedings of the Eye Tracking Research \& Applications Symposium 2000 (pp. 97-103). New York: ACM.

LoschKy, L. C., \& McConKIE, G. W. (2002). Investigating spatial vision and dynamic attentional selection using a gaze-contingent multiresolutional display. Journal of Experimental Psychology: Applied, $\mathbf{8}$ 99-117.

Loschiy, L. C., McConkie, G. W., Yang, J., \& Miller, M. E. (2002). The effects of eccentricity-dependent image filtering on saccade targeting in natural images [Abstract]. Journal of Vision, 2, 170a.

Mackworth, N. H. (1965). Visual noise causes tunnel vision. Psychonomic Science, 3, 67-68.

Parkhurst, D., Culurciello, E., \& Neibur, E. (2000). Evaluating variable resolution displays with visual search: Task performance and eye movements. In A. T. Duchowski (Ed.), Proceedings of the Eye Tracking Research \& Applications Symposium 2000 (pp. 105-109). New York: ACM.

Pomplun, M., Reingold, E. M., \& Shen, J. (2001). Investigating the visual span in comparative search: The effects of task difficulty and divided attention. Cognition, 81, B57-B67.

Reingold, E. M., Loschky, L. C., McConkie, G. W., \& Stampe, D. M. (in press). Gaze-contingent multi-resolutional displays: An integrative review. Human Factors.

ShIOIRI, S., \& IKEDA, M. (1989). Useful resolution for picture perception as a function of eccentricity. Perception, 18, 347-361.

Turner, J. A. (1984). Evaluation of an eye-slaved area-of-interest display for tactical combat simulation. In The 6th Interservice/Industry Training Equipment Conference and Exhibition (pp. 75-86).

van Diepen, P. M. J., \& WAMPERS, M. (1998). Scene exploration with Fourier-filtered peripheral information. Perception, 27, 1141-1151.

Watson, B. A., Walker, N., Hodges, L. F., \& Worden, A. (1997). Managing level of detail through peripheral degradation: Effects on search performance with a head-mounted display. ACM Transactions on Computer-Human Interaction, 4, 323-346.

WiLliams, L. J. (1985). Tunnel vision induced by a foveal load manipulation. Human Factors, 27, 221-227.

WiLliams, L. J. (1988). Tunnel vision or general interference? Cognitive load and attentional bias are both important. American Journal of Psychology, 101, 171-191.

WiLliams, L. J. (1989). Foveal load affects the functional field of view. Human Performance, 2, 1-28.

YANG, J., CoIA, T., \& Miller, M. (2001). Subjective evaluation of retinaldependent image degradations. In PICS 2001: Image Processing, Image Quality, Image Capture Systems Conference (pp. 142-147) Springfield, VA: Society for Imaging Science and Technology. 